\title{
Vibration Reduction Performance of Damping-Enhanced Water-Lubricated Bearing Using Fluid-Saturated Perforated Slabs
}

\author{
Yong $\operatorname{Jin}^{1,2^{*}} \mathbb{D}$, Jianjun Lu², Wu Ouyang ${ }^{2}$, Zhenglin Liu ${ }^{2}$ and Kunsheng Lao ${ }^{2}$
}

\begin{abstract}
As the first link element for the transmission of shaft vibration to the pedestal and even to the hull, water-lubricated bearing plays a key role in suppressing vibration. Although the porous structure is considered as one of the main methods for improving the wideband vibration and noise reduction performance of materials in many industrial fields, the studies in the field of water-lubricated bearing remain insufficient. To enhance vibration reduction performance, a fluid-saturated perforated slab is designed in this study, and via the establishment of a fluid-solid coupled vibration model, the influence law and impact levels were analyzed and verified by simulation and experiments. The results obtained verified that the total vibration amplitude of damping-enhanced stern bearing in the vertical direction was smaller than that of the normal stern bearing, and the reduction amplitude of the characteristic frequency agreed with the optimal value at approximately 0.1 of the volume fraction of the liquid phase when the solid-fluid phase was rubber-water. Additionally, the increase in fluid fraction did not enhance the damping effect, instead, it unexpectedly reduced the natural frequency of the raw material significantly. This research indicates that the design of the fluid-saturated perforated slab is effective in reducing the transmission of the vibration amplitude from the shaft, and presents the best volume fraction of the liquid phase.
\end{abstract}

Keywords: Water-lubricated stern bearing, Fluid-saturated perforated slabs, Fluid-solid coupled vibration model

\section{Introduction}

Underwater vehicles are commonly subjected to several kinds of vibrations and noise during navigation owing to the installation of propulsion shafting and the friction between parts in contact. Additionally, as the first element of the transmission of vibration from shaft to hull and operating part in severe conditions, stern bearing plays a vital role in the attenuation of vibration. Presently, the type of stern bearing applied to vibration and noise reduction by most researches is the conventional bearing structure, as illustrated in Figure 1; and an important way of reducing vibration and noise is by enhancing the damping performance of the bearing, improving

\footnotetext{
*Correspondence: jy761121@whut.edu.cn

${ }^{1}$ Ministry of Education, Key Laboratory of High Performance Ship

Technology (Wuhan University of Technology), Wuhan 430063, China Full list of author information is available at the end of the article
}

the material components of the bearing slab, or adding a damping layer. Based on slippery polymer alloy (SPA) materials, Goodrich [1], a US company, cooperated with Duramax Marine to develop a new type of polymer alloy water-lubricated bearing that has achieved optimal optimization effects on the carrying capacity, reduced friction coefficients and wear rates, and can adapt seamlessly to environments with heavy load condition and high sediment content. Peng et al. [2] introduced nanoscale $\mathrm{ZnOw}$ whiskers to the base of the traditional bearing material formula, and the test results indicated that the mechanical and tribological properties of the bearing were improved by adopting the strengthening effect. Kuang et al. [3], Huang et al. [4], and Chang et al. [5] have also conducted similar studies on bearings, and they reached the consistent conclusion that nano-composites could improve friction and wear properties. 


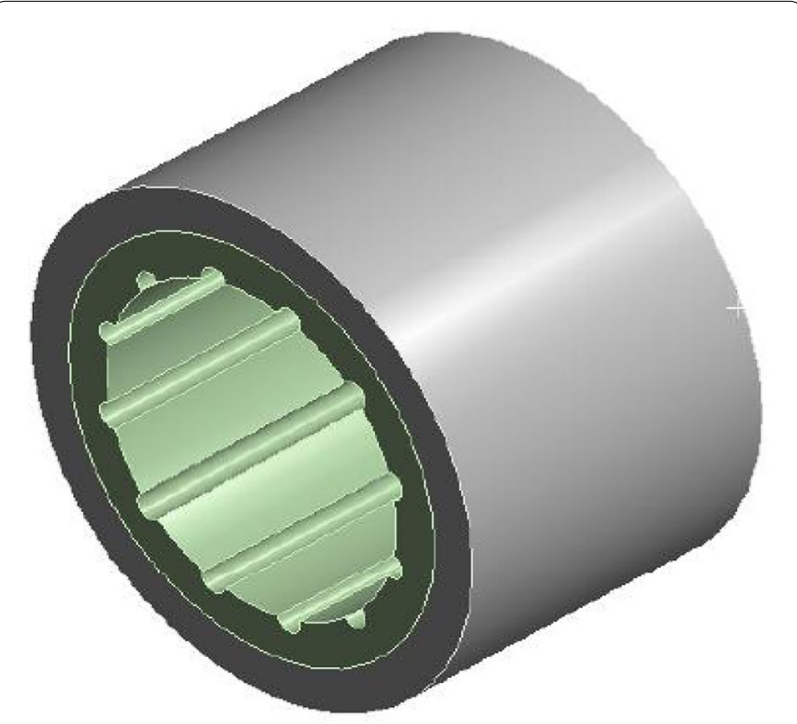

Figure 1 Normal water-lubricated bearing

Another method for improving the lubrication performance of the bearing is by changing the distribution of the slabs and padding the surface texturing to reduce the friction coefficient and indirectly reduce the vibration generated in the operation shafting process. Li et al. [6] adopted the complex mode analysis method to consider the influence law of the thickness, width, and number of the bearing slabs on friction noise frequency, and the results showed that with the lath-arranged type, increasing the thickness and number of laths, as well as decreasing its width, could effectively reduce the possibility of frictional noise and the strength of the noise. Tala-Ighil et al. [7, 8] pointed out that the texturing over the entire bearing surface did not necessarily improve the bearings' performance and the complete texturing could generate some positive effects for low rotational speeds, whereas a partial texturing at the outlet of the active pressure zone had a more significant effect. Blatter et al. [9] experimentally studied the effect of the micro-grooves on sliding friction using a pin-on-disk tribometer, and suggested that wear could be reduced and the sliding life significantly extended by an appropriate size and form of the microgrooves. Litwin [10] compared two types of waterlubricated bearings via experiments, and the results obtained indicated that the three-layer sliding bearing with lubrication grooves in the upper part of the bush possessed optimal tribological properties. Bhardwaj et al. [11], Dong et al. [12], Sudeep [13], Yamada [14], Adamczak et al. [15], and Yang et al. [16] also carried out studies on the effect of texture on the vibration in various bearings, and obtained positive results.
Although all these improvements have been applied to engineering practices, to improve the water-lubricated bearing performance of vibration and noise reduction, it is necessary to carry out innovative thinking and explore new technologies and methods. In recent years, the porous structure method has been considered as an important method for improving the performance of material vibration and noise reduction. Okada et al. [17] explored and tested a novel type of copper-graphite brush using the porous structure method, and the results obtained demonstrated that the application of this new porous brush does not only particially reduce the vibration caused by sliding friction, but also correspondingly reduces the friction coefficient, wear rate, and contact voltage. By comparing the natural frequency of stress deformation and modal analysis of porous and non-porous vertical columns, Shan et al. [18] concluded that porous vertical columns did not only satisfy the basic performance index, but could also preserve materials, thus reducing the weight of the structure and improving the lifting rate performance of the machining center. Zhou et al. [19] combined the random vibration and non-compressible porous elastic medium theories to develop a porous elastic plate containing saturated liquids, and discussed the influence of fluid-solid coupling on the displacement and bending moment of the porous elastic plate. The results obtained showed that the permeability coefficient of the saturated liquid in the elastic plates could be altered to reduce the random vibration of the plates. Rajesh et al. [20] studied the impact of several arbitrary porous structure on the performance of the step bearing lubricated with a magnetic fluid, and suggested a porous squeeze step bearing design with globular spheres in the porous region, considering a variable magnetic field strength of approximately $104 \mathrm{~A} / \mathrm{m}$. Eder et al. [21] characterized the tribological behavior and wear resistance of porous journal bearing systems operating under heavy loads and at small rotational speeds, and propsed methods to visualize and quantify the wear of bearings.

Nevertheless, in addition to oil-lubricated bearing, aerostatic bearings have employed the pore structure method to improve the performance of lubrication performance [22-29]. However, the application research of water-lubricated stern bearing design, using the porous structure method to reduce vibration and noise, has not been carried out in depth. Regarding the porous medium theory, a damping-enhanced water-lubricated bearing with fluid-saturated perforated slabs was designed, as illustrated in Figure 2. By developing the fluid-solid coupled vibration model, the influence law and impact level of the fluid-saturated perforated structure design on the 

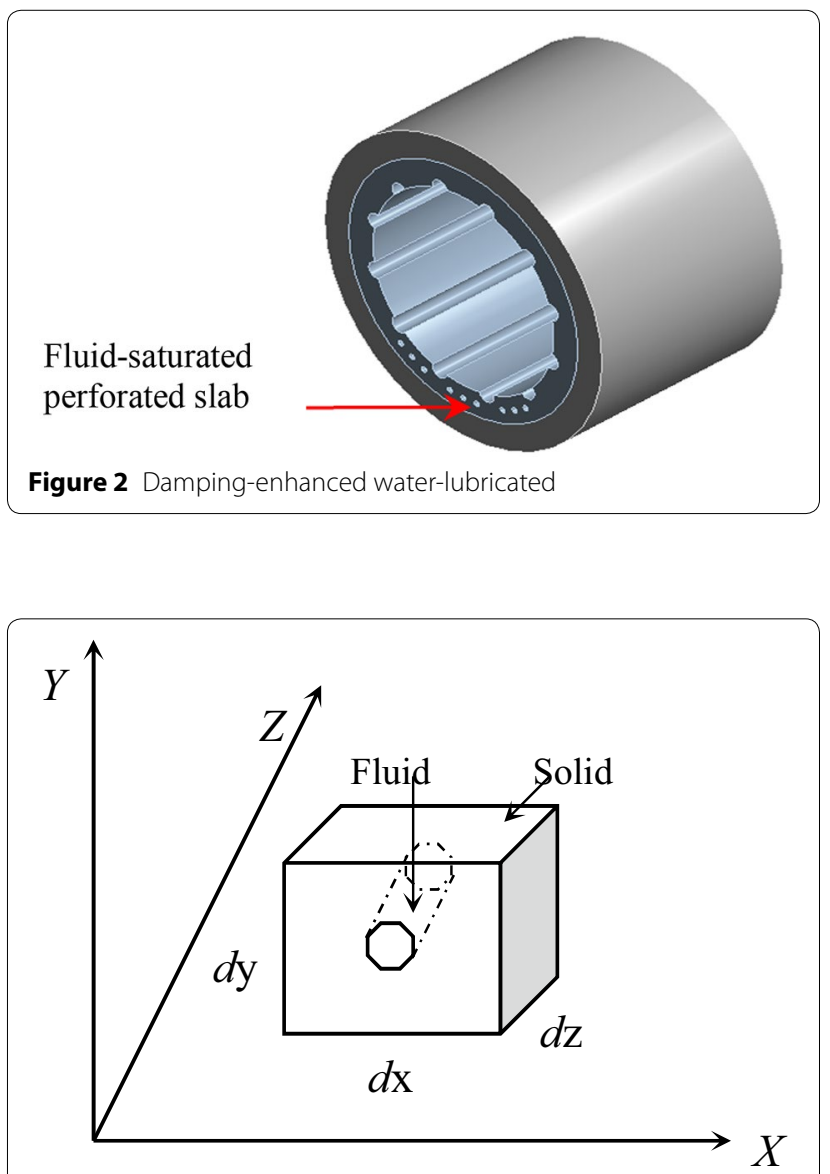

Figure 3 Element model

integral structure of water-lubricated stern bearing were analyzed, and the effect of vibration and noise reduction on the structural design was verified via experiments.

\section{Analysis}

\subsection{Fundamental Equation}

In the actual operation process, the attenuation mode of the external excitation acting on the surface of the slab is consistent in its direction, however, the pressure gradient of the fluid exists in the axial direction while the difference in the bearing pressure of the slab exists in the circumferential direction. Therefore, as shown in Figure 3, a unit body containing one hole could be obtained from the slat structure for analysis, and the equation of motion equilibrium for fluid-solid coupled two-phase media could be derived via the Lagrangian generalized coordinate system.

Given that $u=\left\{u_{x}, u_{y}, u_{z}\right\}$ and $U=\left\{U_{x}, U_{y}, U_{z}\right\}$ are the generalized Lagrangian coordinates of the solid and fluid phases, respectively, both phases undergo an insignificant deformation. In the loading process, the boundary of the solid-fluid phase remains continuous without mass exchange, and the kinetic energy of the unit body is expressed as:

$$
T=\frac{1}{2} \rho_{\mathrm{s}} n_{\mathrm{s}}\left(\dot{u}_{x}^{2}+\dot{u}_{y}^{2}+\dot{u}_{z}^{2}\right)+\frac{1}{2} \rho_{\mathrm{f}} n_{\mathrm{f}}\left(\dot{U}_{x}^{2}+\dot{U}_{y}^{2}+\dot{U}_{z}^{2}\right),
$$

where $\rho_{\mathrm{s}}, n_{\mathrm{s}}, \rho_{\mathrm{f}}$, and $n_{\mathrm{f}}$ represent the density of the solid phase material, solid volume fraction, density of the fluid phase material, and fluid volume fraction, respectively. The solid and fluid phases of the unit satisfy the relationship $n_{\mathrm{s}}+n_{\mathrm{f}}=1$.

The slab material is an isotropic polymer material, and the potential energy is considered an elastic potential energy. The saturated fluid in the hole is a Newtonian fluid, such as water or silicon oil, etc., and according to the fluid dynamics theory, the potential energy considers the positional and pressure potential energies. Therefore, the total potential energy of the unit body is expressed as:

$$
\begin{aligned}
V= & \frac{1}{2} k\left(u_{x}^{2}+u_{y}^{2}+u_{z}^{2}\right)+\left(U_{x}+U_{y}+U_{z}\right) \\
& +\left(\frac{P_{\mathrm{f} x}}{\rho_{f} g}+\frac{P_{\mathrm{f} y}}{\rho_{f} g}+\frac{P_{\mathrm{f} z}}{\rho_{f} g}\right) .
\end{aligned}
$$

In Eq. (2), the first, second, and third parts represent the solid elastic potential energy, fluid phase positional potential energy, and pressure potential energy of the fluid phase, respectively, whereas $k$ is the stiffness. Additionally, $P_{\mathrm{f} x}, P_{\mathrm{f} y}$, and $P_{\mathrm{f} z}$ represent the pressure of the fluid phase in three different directions.

The energy dissipation system of the unit body mainly consists of the internal damping of both solid and fluid phases, including the frictional damping generated by the relative motion between the contact surfaces of the solid and fluid. Therefore, the energy dissipation function of the unit body can be expressed as:

$$
\left\{\begin{array}{l}
D_{\mathrm{s}}=\frac{1}{2} C_{\mathrm{s}}\left(\dot{u}_{x}^{2}+\dot{u}_{y}^{2}+\dot{u}_{z}^{2}\right), \\
D_{\mathrm{ff}}=\frac{1}{2} C_{\mathrm{ff}}\left(\dot{U}_{x}^{2}+\dot{U}_{y}^{2}+\dot{U}_{z}^{2}\right), \\
D_{\mathrm{fs}}=\frac{1}{2} C_{\mathrm{fs}}\left(\left(\dot{u}_{x}-\dot{u}_{x}\right)^{2}+\left(\dot{U}_{y}-\dot{u}_{y}\right)^{2}+\left(\dot{U}_{z}-\dot{u}_{z}\right)^{2}\right] \\
D=D_{\mathrm{s}}+D_{\mathrm{ff}}+D_{\mathrm{fs}},
\end{array}\right.
$$

where $D_{\mathrm{s}}, C_{\mathrm{s}}, D_{\mathrm{ff}}, C_{\mathrm{ff}}, D_{\mathrm{fs}}$, and $C_{\mathrm{fs}}$ represent the inner damping of the solid phase, inner damping coefficient of the solid phase, inner damping of the fluid phase, inner damping coefficient of the liquid phase, frictional damping between the solid and fluid phases, and frictional damping coefficient between the solid and fluid phases, respectively.

In the generalized coordinate system, the Lagrangian motion equation of the vibration system is generally expressed as: 


$$
\frac{\mathrm{d}}{\mathrm{d} t}\left(\frac{\partial T}{\partial \dot{q}_{i}}\right)-\frac{\partial T}{\partial q_{i}}+\frac{\partial V}{\partial q_{i}}+\frac{\partial D}{\partial \dot{q}_{i}}=Q_{i}, \quad(i=1,2,3, \ldots, n)
$$

where $T, V, D, q, Q$, and $i$ represent the system's kinetic energy, its potential energy, dissipative energy function, generalized coordinate, non-conservative generalized force, and degree of freedom, respectively.

Based on Eq. (4), we first obtained the derivative of the $u_{\mathrm{x}}$ coordinate of the solid phase for Eqs. (1), (2), and (3), from which we then derived the following equations:

$$
\begin{aligned}
& \frac{\mathrm{d}}{\mathrm{d} t}\left(\frac{\partial T}{\partial \dot{u}_{x}}\right)=\rho_{\mathrm{s}} n_{\mathrm{s}} \ddot{u}_{x}, \frac{\partial T}{\partial u_{x}}=0, \\
& \frac{\partial V}{\partial u_{x}}=k u_{x}, \frac{\partial D}{\partial \dot{u}_{x}}=\left(C_{\mathrm{s}}+C_{\mathrm{fs}}\right) \dot{u}_{x}-C_{\mathrm{fs}} \dot{u}_{x} .
\end{aligned}
$$

By substituting the above into Eq. (4), the balance equation of the $u_{x}$-coordinate direction can be obtained from the following equation:

$$
\rho_{\mathrm{s}} n_{\mathrm{s}} \ddot{u}_{x}+\left(C_{\mathrm{s}}+C_{\mathrm{fs}}\right) \dot{u}_{x}-C_{\mathrm{fs}} \dot{u}_{x}+k u_{x}=0 .
$$

In the same way, the vibration equilibrium equation of solid phase in the $u_{y}$ and $u_{z}$ directions can also be obtained.

Then, taking the derivative of the $U_{x}$ coordinate of fluid phase, the following equation can be obtained:

$$
\begin{aligned}
& \frac{\mathrm{d}}{\mathrm{d} t}\left(\frac{\partial T}{\partial \dot{U}_{x}}\right)=\rho_{\mathrm{f}} n_{\mathrm{f}} \ddot{U}_{x}, \frac{\partial T}{\partial U_{x}}=0, \\
& \frac{\partial D}{\partial \dot{U}_{x}}=\left(C_{\mathrm{ff}}+C_{\mathrm{fs}}\right) \dot{U}_{x}-C_{\mathrm{fs}} \dot{U}_{x}, \frac{\partial V}{\partial U_{x}}=1+\frac{1}{\rho_{f} g} \frac{\partial P_{x}}{\partial U_{x}},
\end{aligned}
$$

where $\frac{\partial P_{x}}{\partial U_{x}}$ represents the pressure gradient of the fluid phase in the $U_{x}$ direction. Additionally, because the stress of the fluid is equal to the pressure value and opposite to its direction, it can be derived according to Newton's law and expressed as:

$$
\frac{\partial P_{x}}{\partial U_{x}}=-\rho_{\mathrm{f}} \ddot{U}_{x}^{2} .
$$

Ignoring the second-order small quantity owing to negligible deformation, the balance equation of the $U_{x}$-coordinate direction in the liquid phase can be obtained from the equation expressed as:

$$
\rho_{\mathrm{f}} n_{\mathrm{f}} \ddot{U}_{x}+\left(C_{\mathrm{ff}}+C_{\mathrm{fs}}\right) \dot{U}_{x}-C_{\mathrm{fs}} \dot{u}_{x}+1=0 .
$$

Similarly, the vibration equilibrium equation of the fluid phase in the $U_{y}$ and $U_{z}$ directions can be obtained.
By arranging the balanced equations in the six degrees of freedom direction, the second-order differential equations of the vibration equilibrium of the unit body can be obtained:

$$
\boldsymbol{M} \cdot\left\{\begin{array}{c}
\ddot{u} \\
\ddot{u}
\end{array}\right\}+\boldsymbol{C} \cdot\left\{\begin{array}{c}
\dot{u} \\
\dot{U}
\end{array}\right\}+\boldsymbol{K} \cdot\left\{\begin{array}{c}
u \\
U
\end{array}\right\}=\boldsymbol{b},
$$

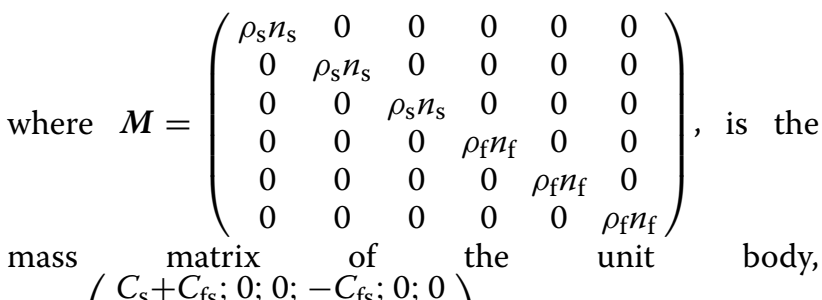
$C=\left(\begin{array}{c}C_{\mathrm{s}}+C_{\mathrm{fs}}, 0,0,-C_{\mathrm{fs}} ; 0 ; 0 ;-C_{\mathrm{fs}} ; 0 \\ 0 ; 0 ; C_{\mathrm{s}}+C_{\mathrm{ff}} ; 0 ; 0 ;-C_{\mathrm{fs}} \\ -C_{\mathrm{fs}} ; 0 ; 0 ; C_{\mathrm{ff}}+C_{\mathrm{fs}} ; 0 ; 0 \\ 0 ;-C_{\mathrm{fs}} ; 0 ; 0 ; C_{\mathrm{ff}}+C_{\mathrm{fs}} ; 0 \\ 0 ; 0 ;-C_{\mathrm{fs}} ; 0 ; 0 ; C_{\mathrm{ff}}+C_{\mathrm{fs}}\end{array}\right)$, is the damping matrix of the unit body; $K=\left(\begin{array}{cccccc}k & 0 & 0 & 0 & 0 & 0 \\ 0 & k & 0 & 0 & 0 & 0 \\ 0 & 0 & k & 0 & 0 & 0 \\ 0 & 0 & 0 & 0 & 0 & 0 \\ 0 & 0 & 0 & 0 & 0 & 0 \\ 0 & 0 & 0 & 0 & 0 & 0\end{array}\right)$, is the stiffness matrix of the unit body; $\boldsymbol{b}=\{0,0,0,-1,-1,-1\}$, is the load vector.

\subsection{Fundamental Equation}

In general, it is difficult to determine an analytic solution for the second-order differential equations. Therefore, to simulate the vibration process of the unit, the initial conditions under a negligible deformation could be express as:

$$
\begin{aligned}
& \left\{u_{x}, u_{y}, u_{z}\right\}=\{0.01,0.01,0.01\}, \\
& \left\{\dot{u}_{x}, \dot{u}_{y}, \dot{u}_{z}\right\}=\{0,0,0\} \\
& \left\{U_{x}, U_{y}, U_{z}\right\}=\{0.01,0.01,0.01\} \\
& \left\{\dot{U}_{x}, \dot{U}_{y}, \dot{U}_{z}\right\}=\{0,0,0\}
\end{aligned}
$$

The solid phase of the unit is a rubber material, whereas the liquid phase is water. The specific characteristic parameters were selected as presented in Table 1 , and after the volume fraction of the liquid phase was set,

Table 1 Parameters of unit body

\begin{tabular}{ll}
\hline Parameter & Value \\
\hline Density of solid phase $\rho_{\mathrm{s}}\left(\mathrm{kg} / \mathrm{m}^{3}\right)$ & 1190 \\
Density of fluid phase $\rho_{\mathrm{f}}\left(\mathrm{kg} / \mathrm{m}^{3}\right)$ & 998 \\
Stiffness coefficient of solid phase $\mathrm{k}(\mathrm{N} / \mathrm{m})$ & $2 \times 10^{8}$ \\
Inner damping coefficient of solid phase $C_{\mathrm{s}}$ & 0.2 \\
Inner damping coefficient of fluid phase $C_{\mathrm{ff}}$ & 0.02 \\
Friction damping coefficient between fluid-solid phase $C_{\mathrm{fs}}$ & 0.4
\end{tabular}


Table 2 Parameters of unit body

\begin{tabular}{ll}
\hline Parameter & Value \\
\hline Diameter of inner-pore $(\mathrm{mm})$ & 4 \\
Geometric parameters of the top surface (length $\times$ width) $(\mathrm{mm})$ & $12 \times 15$ \\
Bottom radian $\left(^{\circ}\right)$ & 10 \\
High $(\mathrm{mm})$ & 15 \\
\hline
\end{tabular}

the results of the numerical analysis was obtained using the ODE45 function of Matlab libraries.

\subsection{Numerical Simulation}

To further analyze the influence of fluid-saturated perforated slab on the vibration reduction performance of bearing, the finite element analysis tool was used to compare the transient response amplitude of the conventional bearing and fluid-saturated perforated slabs under the same load. Additionally, similar to Section 2.2, to reduce the load of the numeric simulation and number of meshes, a unit body of slat with a single hole was intercepted in the bottom of the bearing.

The solid part of the unit is a rubber material, whereas the fluid part is water. The specific characteristic parameters are selected as presented in Table 2. The grid size of the solid part is set to $1 \mathrm{~mm}$, and the inner-pore surface of the perforated slab is set to the refinement of 2 to improve the data mapping of the fluid-solid coupling interface, whereas the fluid part of the perforated slab is a meshing with a size of $0.1 \mathrm{~mm}$. Because the slab has axial similarity, the meshing method adopts sweep mode, hence, the diagram of the resulting meshing model is shown in Figure 4.

Fixed Support is applied to the underside of both slat units because the slats are installed in the outer bushing, with a $0.02 \mathrm{MPa}$ pressure load on the top surface of the unit. The inner-pore surface of the perforated slab is set as a fluid-solid interface. The state of the fluid domain is set as turbulence, and the k-epsilon model is applied. The pressure is set on both sides of fluid domain, with pressures of 0.02 and $0 \mathrm{MPa}$ at the input and output, respectively.

The transient response analysis of the normal bearing slab can be conducted by directly using the transient structural module of the ANSYS Workbench, but the transient analysis based on fluid-structure interaction requires the establishment of bi-directional fluid-structure interaction simulations between the ANSYS fluent module and transient structural module via the system coupling module. The pressure values in the fluid domain were calculated and transferred to the solid domain, then the pressure changes deformed the inner pores of the rubber layer, thus influencing the pressure distribution,

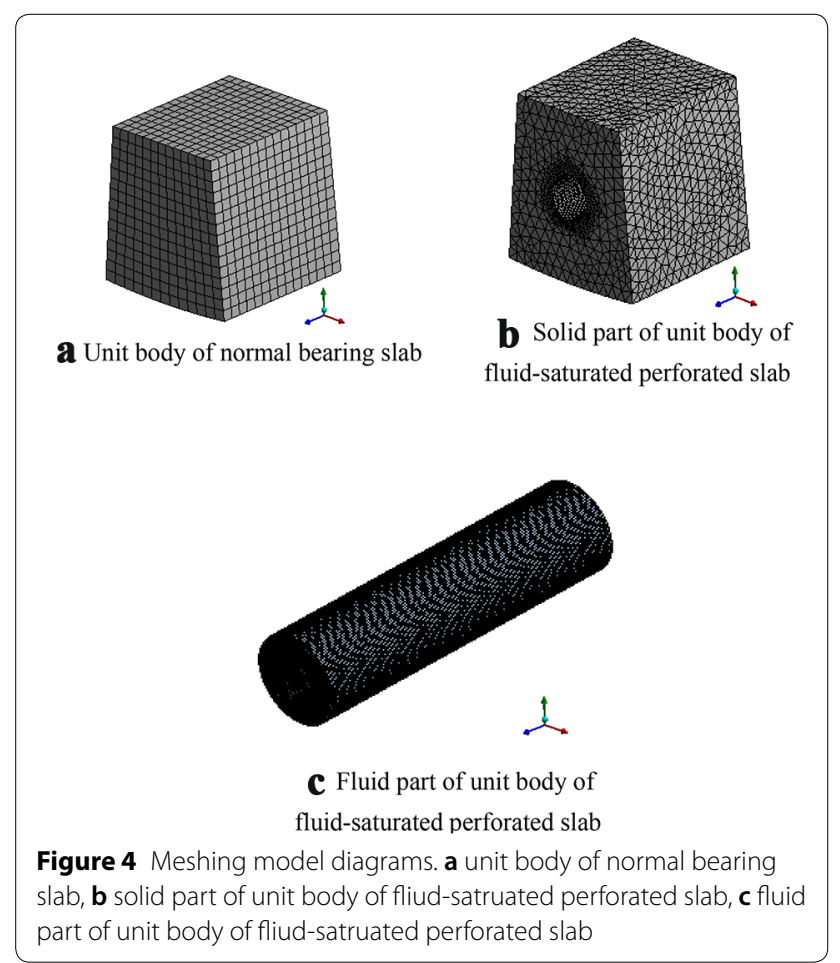

before it is transmitted back to the fluid domain. By applying continuous iterative operations, the steady distribution on the fluid-solid interface was finally achieved.

\subsection{Experiments}

We sought to further verify the positive effect of the fluidsaturated perforated structure on the vibration and noise reduction of stern bearing. Vibration tests were carried out for the normal bearing and damping-enhanced bearing with fluid-saturated perforated slabs with a diameter of $4 \mathrm{~mm}$ to study the vibration variation of the stern bearing under different speeds, with or without perforated slabs. The vibration test was carried out on the SSB-100 stern shaft testing bench of the Wuhan University of Technology, and the test bench was mainly composed of driving, test, and loading parts. The spindle of the bench has an outside diameter of approximately $150 \mathrm{~mm}$ on the journal. The loading method, which is an intermediate radial loading, was employed to ensure the uniformity of the bearing pressure. The vibration amplitude signals are collected by two acceleration sensors arranged on the test bearing seat in the horizontal and vertical directions. The partial test bench and sensor installation schematic are shown in Figure 5.

The outer diameter of the test bearing is $185 \mathrm{~mm}$, with an inner diameter of $150 \mathrm{~mm}$ and length-diameter ratio of approximately $1: 1$, which is the bearing size of bench. The damping-enhanced bearing adopts the same material and 


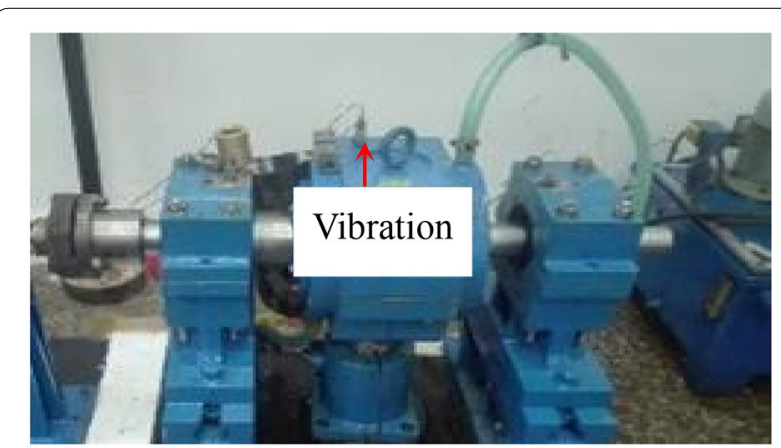

Figure 5 Schematic of tested bearing part of the test platform and sensors installation

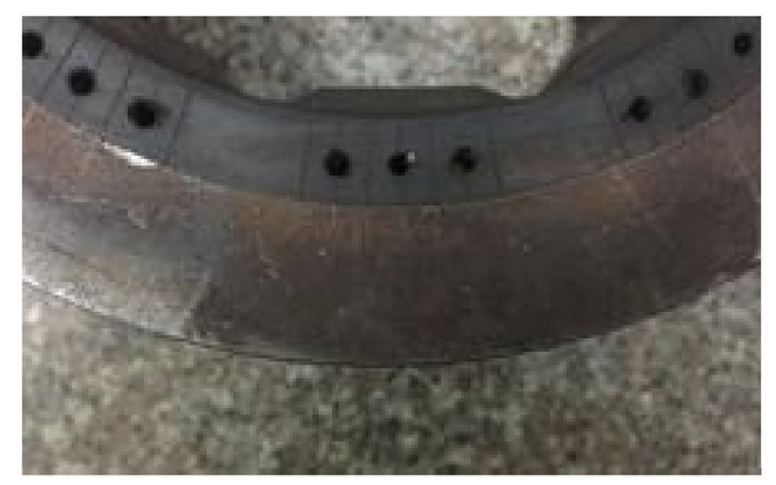

Figure 6 Damping-enhanced bearing with fluid-saturated perforated slabs

tank arrangement as the normal bearing, with the difference of the three 4-mm uniform through-holes in several slabs of the bottom load-bearing area, as shown in Figure 6. Additionally, when the circulation lubrication water channel is opened, the pressured water will pass through and fill these holes. The normal bearing adopts the same structure as the damping-enhanced bearing without the holes.

In the test, the temperature of the lubricating cooling water was at $25{ }^{\circ} \mathrm{C}$ and the flow rate was $20 \mathrm{~L} / \mathrm{min}$. A constant vertical load was applied to the test stern bearing via the hydraulic loading system, which was set at 0.4 $\mathrm{MPa}$. The test speeds were set at 40, 80, 120, 160 and 200 $\mathrm{r} /$ min consecutively as they gradually increased from low to high speed. After each working condition was stable, the vibration acceleration signals of $15 \mathrm{~s}$ were recorded via the $B \& K$ analysis system.

\section{Results and Discussion}

\subsection{Volume Fraction of the Liquid Phase}

The volume fractions of the liquid phase were set as 0 , $0.1,0.2,0.4$ and 0.6 to compare the vibration characteristics of the unit body with holes or without holes. At $\Delta t$
$=0.01$, the movements of the unit body within $1 \mathrm{~s}$ can be obtained, and the results of the numerical analysis are shown in Figure 7.

Comparing these time domain graphs, as shown in Figure $7 \mathrm{a}-\mathrm{e}$, the damping effect of pore-fluid on the motion of the unit body is not significant as no obvious vibration amplitude reduced, except the decreasing free-vibration frequency. However, on the frequency domain graph, it is evident that the amplitude at the natural frequency varies with the volume fraction of the liquid phase and has the best damping effect, which is decreased by $30 \%$ at approximately 0.1 . This indicates that the low friction damping between the solid and liquid phase, caused by the low viscosity of the liquid in the hole, is slow to attenuate the overall motion form of free vibration, but on the amplitude of the characteristic frequency it already had a significant effect. Besides, for every coupling material of the solid-fluid phase, an optimal volume fraction of the liquid phase may exist to enhance the damping of the whole structure.

\subsection{Influence of Inner-Pore on the Transient Response}

To clearly compare the influence of inner-pore on the transient response, a path was selected in both of the two units, as shown in Figure 8, and the transient response along the path was illustrated in Figure 9.

By comparing the transient response of two units, it can be determined that the displacement of the perforated slab unit is lower than that of the normal bearing slab along the path under the same transient load, which is presented to be a more uniform response in the pore area.

\subsection{Test Results}

Figure 10 presents a waterfall diagram illustrating the variation of vibration amplitude of the normal bearing with rotating speeds in the horizontal and vertical directions.

Figure 11 presents a waterfall diagram showing the vibration amplitude of the damping-enhanced bearing with rotating speeds in the horizontal and vertical directions.

By comparing and analyzing the horizontal vibration acceleration of normal stern bearing (Figure 10(a)) and that of the damping- enhanced stern bearing (Figure 11(a)), it can be determined that the amplitude of vibration acceleration of two kinds of stern bearing mainly occurred in a frequency range of 100-400 Hz, and these amplitudes both increased and the range was widened with the increase in the test shaft speed. However, within this frequency range the vibration amplitude of the damping-enhanced stern bearing 


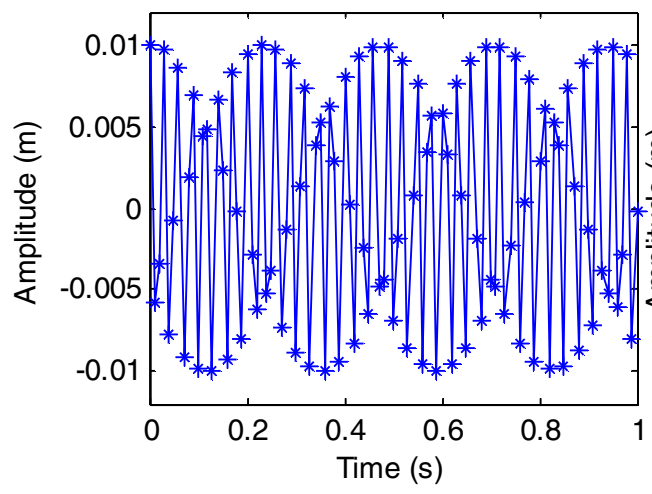

a Time domain graph $\left(n_{\mathrm{f}}=0\right)$

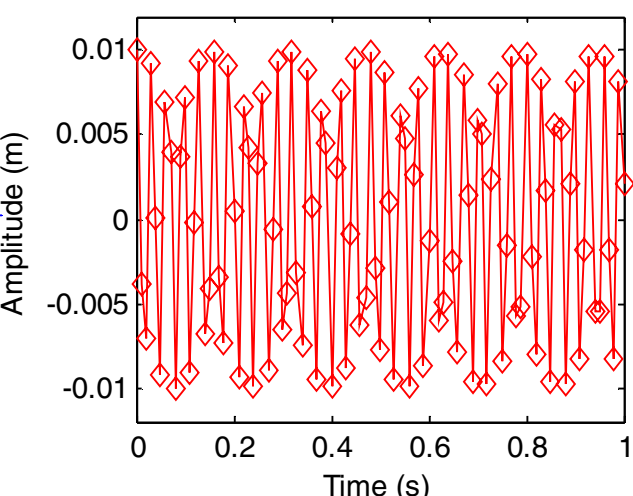

b Time domain graph $\left(n_{\mathrm{f}}=0.1\right)$

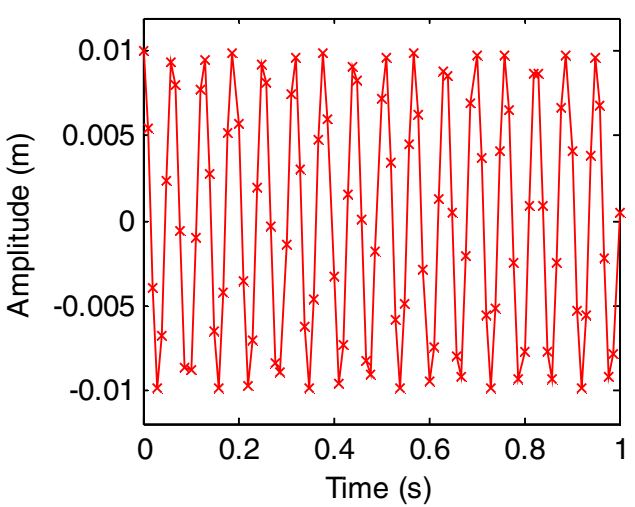

d Time domain graph $\left(n_{\mathrm{f}}=0.4\right)$

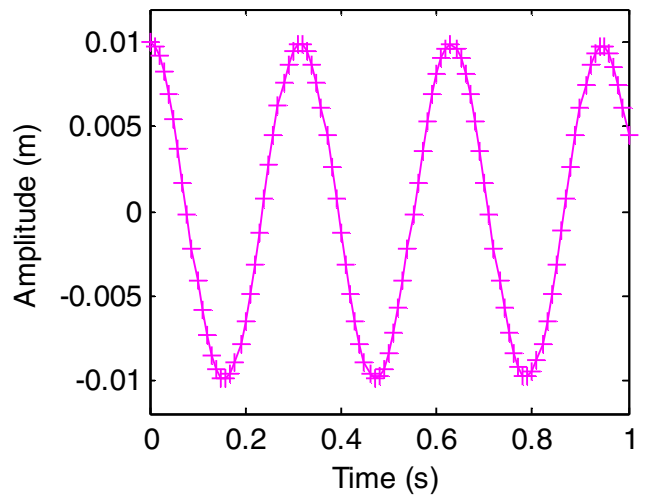

e Time domain graph $\left(n_{\mathrm{f}}=0.6\right)$

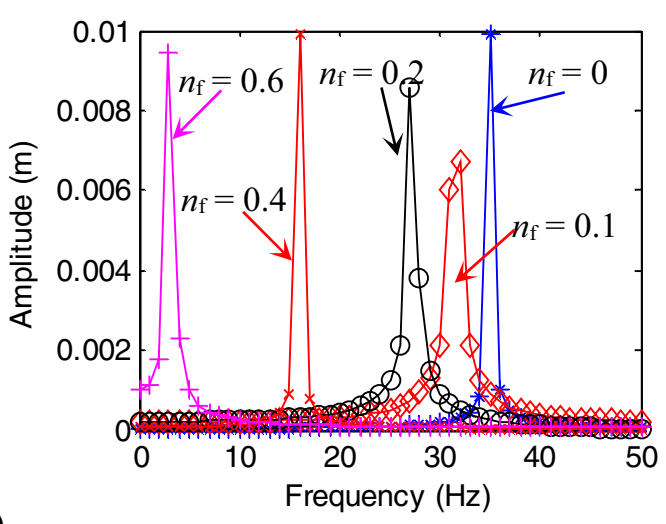

f Frequency domain graph

Figure 7 Motion of unit body. a Time domain graph $(n f=0)$, $\mathbf{b}$ Time domain graph $(n f=0.1)$, e Time domain graph $(n f=0.6), \mathbf{f}$ Frequency domain graph 

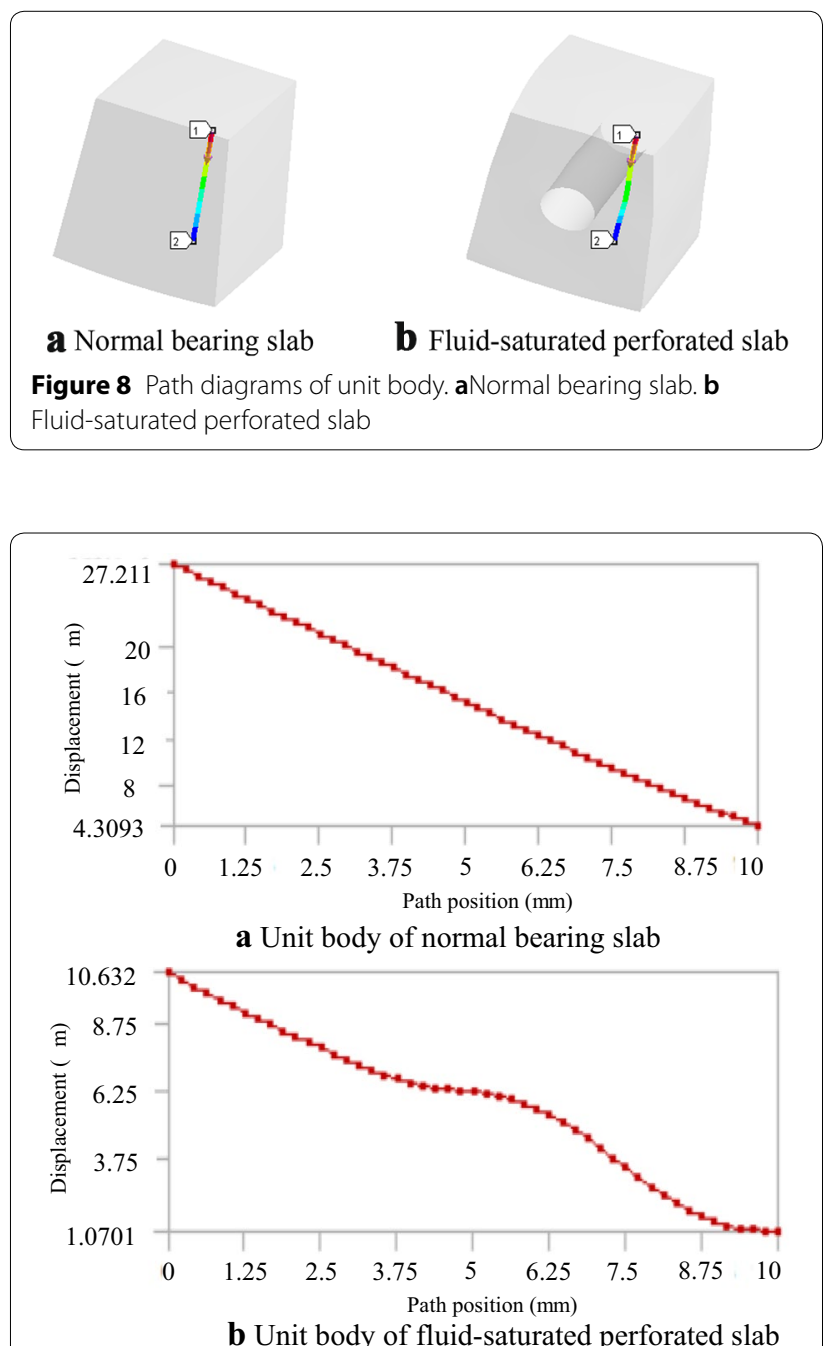

Figure 9 Transient response along the path a Unit body of normal bearing slab $\mathbf{b}$ Unit body of fluid-saturated perforated slab

was less significant than that of normal stern bearing. Meanwhile, by comparing and analyzing the vertical vibration acceleration of normal stern bearing (Figure $10(\mathrm{~b}))$ and that of the damping-enhanced stern bearing (Figure 11(b)), it can be determined that the vibration acceleration increased with the increase in test shaft speed. At a frequency of approximately 250 $\mathrm{Hz}$, which is the characteristic frequency of oil cylinder excitation [17], a larger vibration acceleration amplitude appeared on the normal stern bearing than on the damping-enhanced stern bearing. However, in the other frequency range, there was no significant difference between the two stern bearings. These indicated that the design of the fluid-saturated perforated

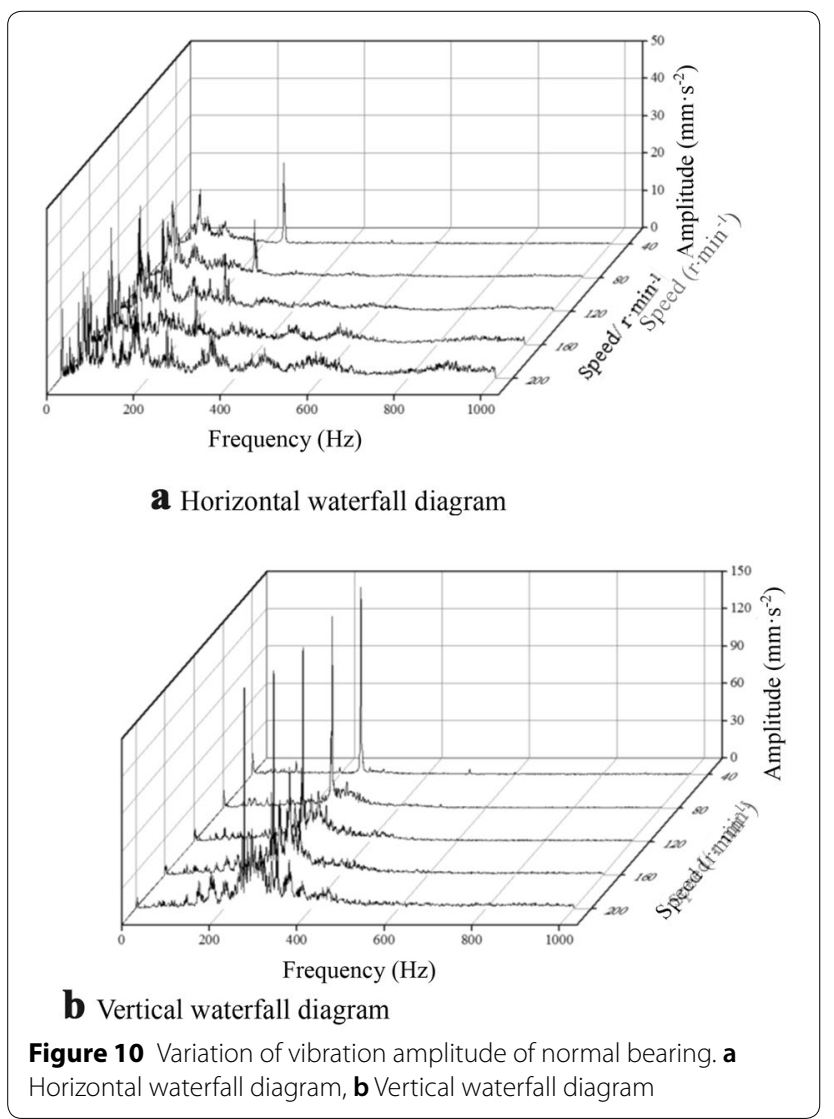

slab structure in the load-bearing area at the bottom of stern bearing can effectively reduce the vibration amplitude generated by running a shaft system.

Under the loading pressure of 0.4 MPa, the total vertical and horizontal vibration values of the normal and damping-enhanced stern bearings within the frequency range of $0-1000 \mathrm{~Hz}$ at different test shaft speeds are presented in Figure 12.

Under the loading pressure condition $0.4 \mathrm{MPa}$, frequency range of $0-1000 \mathrm{~Hz}$, and test shaft rotation speed range of $80-200 \mathrm{r} / \mathrm{min}$, it can be observed from Figure 12(a) that the total vibration amplitude of damping-enhanced stern bearing in the vertical direction was less significant than that of the normal stern bearing, and the total vibration amplitude of the dampingenhanced stern bearing was approximately equal to that of the normal stern bearing in the horizontal direction, as shown in Figure 12(b). This indicated that the vertical vibration attenuation effect is more significant than the horizontal vibration attenuation effect when 


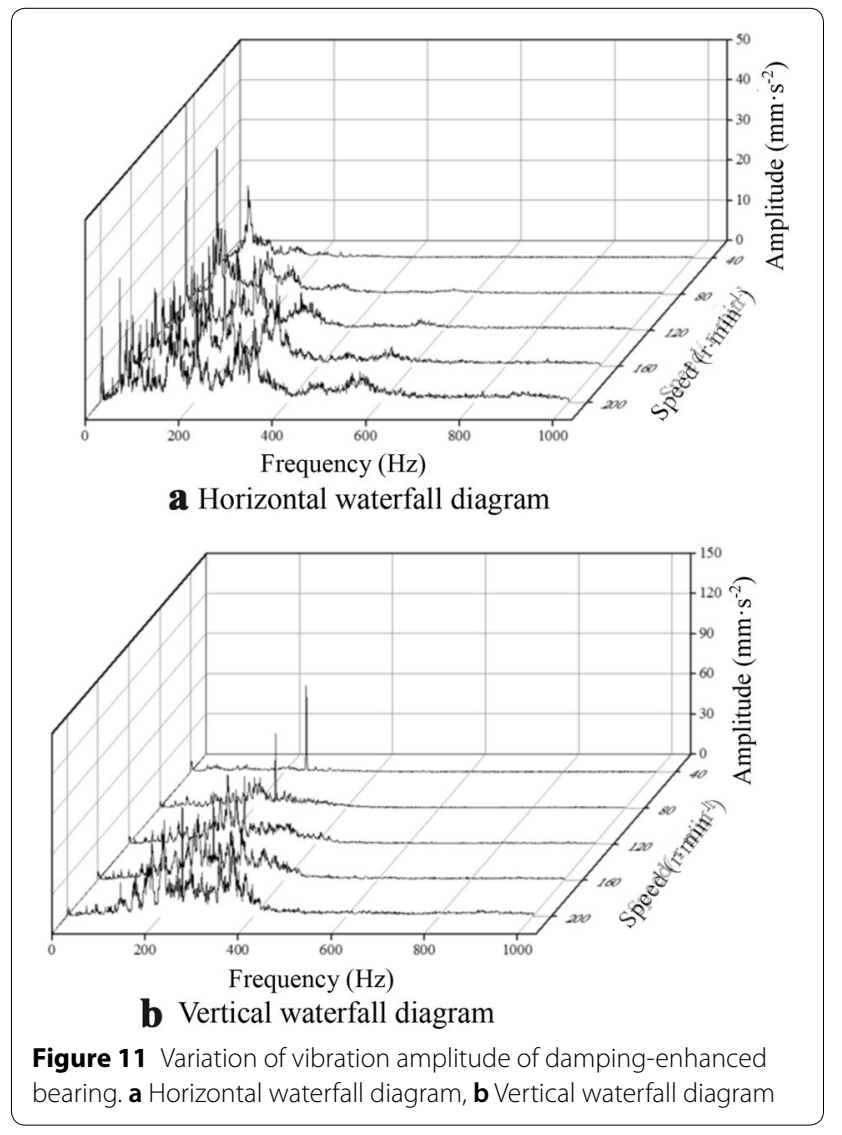

the fluid-saturated perforated design was set in the axial direction of stern bearing slats.

\section{Conclusions}

In conclusion, via the development of the fluid-solid coupled vibration model, the influence law and impact level of the fluid-saturated perforated structure design on the integral structure of the water-lubricated stern bearing were analyzed and verified by simulation and experiment, and the following conclusions were obtained.

(1) The proposed fluid-saturated perforated slab was significantly effective in reducing the vibration amplitude transferred from shaft.

(2) The optimal volume fraction of the liquid phase was obtained to enhance the damping of the whole structure, and when the solid phase material of the bearings was rubber and that of the fluid phase was water, the volume fraction was approximately 0.1. Furthermore, the increase in fluid fraction does not

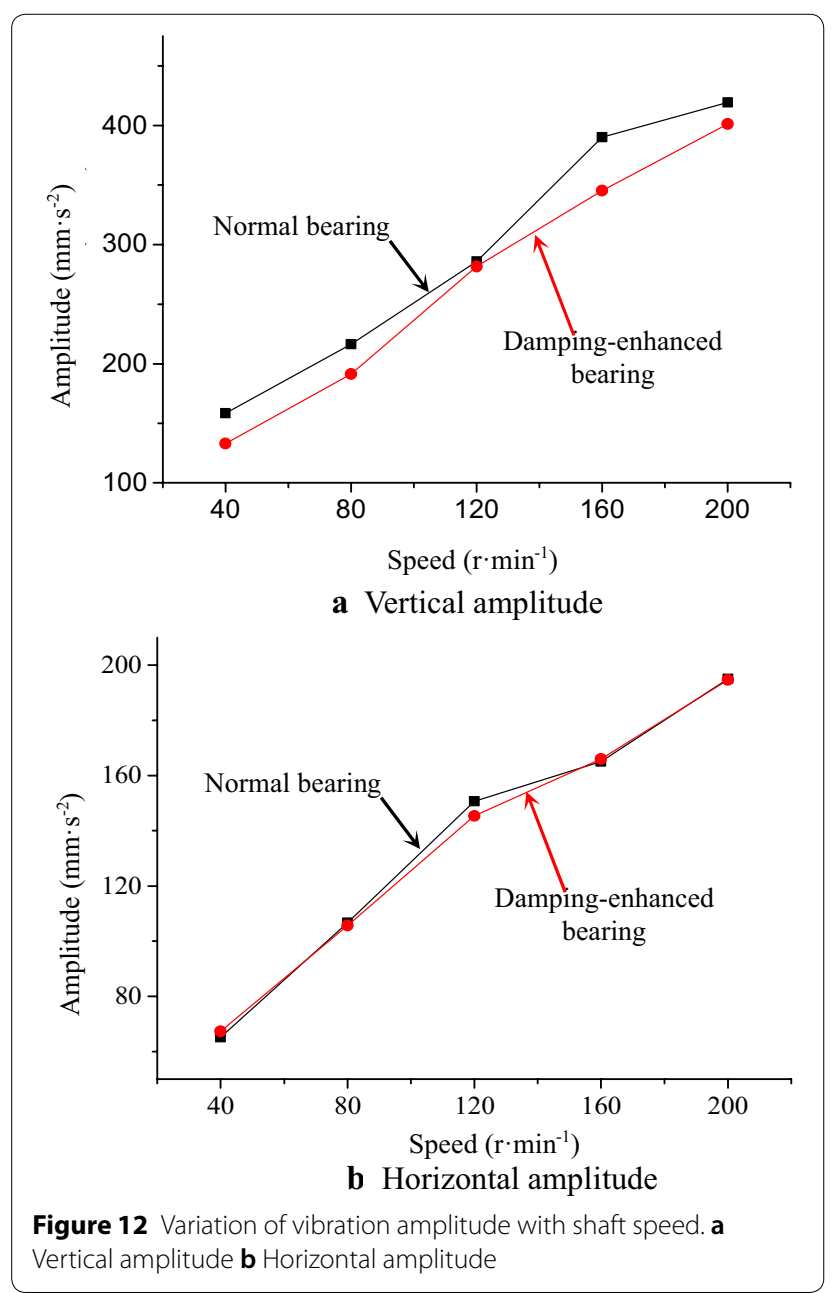

enhance the damping effect, instead, it increases its influence on the natural frequency of the original material.

\section{Acknowledgements}

The authors give sincere thanks to the editors and the reviewers for their patient work and constructive suggestions.

\section{Authors' Contributions}

YJ wrote the initial manuscript; $K L$ assisted with the experimental process; $J$ assisted with the test data collation; WO and ZL revised the manuscript. All authors read and approved the final manuscript.

\section{Authors' information}

Yong Jin, born in 1976, is currently an associate professor at Wuhan University of Technology, China. He received his PhD degree from Wuhan University of Technology, China, in 2016. His research interests include ship shafting engineering and ship enterprise informatization.

Jianjun Lu, born in 1994, is currently a graduate student, majoring in Ship and Marine Engineering, Wuhan University of Technology, China. He is now 
participating in projects at water-lubricated stern bearing group, Wuhan University of Technology, China.

Wu Ouyang, born in 1987, is currently an associate professor at Wuhan University of Technology, China. He received his PhD degree from Xi'an Jiaotong University, China, in 2014. His research interests include rotor dynamics of bearing.

Zhenglin Liu, born in 1949, is currently a professor at Wuhan University of Technology, China. His current research interests are ship shafting engineering.

Kunsheng Lao was born in 1991, and received his master's degree from Wuhan University of Technology, China, in 2017. He is now working in an engine research and development enterprise.

\section{Funding}

Supported by State Key Program Grant of National Natural Science Foundation of China (Grant No. 51579198) and Key Laboratory of High Performance Ship Technology Opening Foundation (Grant No. 2016gxnc04).

\section{Competing interests}

The authors declare no competing financial interests.

\section{Author Details}

${ }^{1}$ Ministry of Education, Key Laboratory of High Performance Ship Technology (Wuhan University of Technology), Wuhan 430063, China. ${ }^{2}$ School of Energy and Power Engineering, Wuhan University of Technology, Wuhan 430063, China.

Received: 13 November 2019 Revised: 10 November 2020 Accepted: 18 November 2020

Published online: 03 December 2020

\section{References}

[1] R L Orndorff, R C Spangler. SPA super demountable bearing: US6648510 B2. 2003-11-18.

[2] J M Peng, J XWang. Research on key technologies to improve bearing capacity of water-lubricated bearing. Journal of Agricultural Machinery, 2005, 36(6): 149-151. (in Chinese)

[3] F M Kuang, X C Zhou, J Huang, et al. Tribological properties of Nitrile Rubber/UHMWPE/Nano-MoS2 water-lubricated bearing material under low speed and heavy duty. Journal of Tribology, 2018, 140(6): 061301.

[4] J Huang, X R Zhou, X Z Zhou, et al. Study on preparation process of a new UHMWPE/graphite/NBR water lubricated bearing material. The 4nd International Conference on Transportation Information and Safety, Canada, 2017: 907-910.

[5] T Chang, C Q Yuan, Z W Guo. Tribological behavior of aged UHMWPE under water-lubricated condition. Tribology International, 2019, 133(1): 1-11.

[6] K Li, J X Wang, Y Guo, et al. Analysis of the impact of slats on friction noise of water-lubricated rubber bearing. Journal of Mechanical Transmission, 2013(11): 138-141. (in Chinese)

[7] Tala-Ighil N, Fillon M. A numerical investigation of both thermal and texturing surface effects on the journal bearings static characteristics. Tribology International, 2015, 90: 228-239.

[8] N Tala-Ighil, M Fillon, P Maspeyrot. Effect of textuted area on the performances of a hydrodynamic journal bearing. Tribology International, 2011, 44: 211-219.

[9] A Blatter, M Maillat, S M Pimenov, et al. Lubricated sliding performance of laser-patterned sapphire. Wear, 1999, 232: 226-230.

[10] Wojciech Litwin. Experimental research on water lubricated three layer sliding bearing with lubrication grooves in the upper part of the bush and its comparison with a rubber bearing. Tribology International, 2015, 82(A): 153-161.
[11] V Bhardwaj, R K Pandey, V K Agarwal. Performance studies of textured race ball bearing. Industrial Lubrication and Tribology, 2019, 71(9): 1116-1124.

[12] J Dong, X J Wang, J Zhang, et al. An experimental research on the vibration of surface-textured journal bearings. Shock \& Vibration, 2017: 1-9.

[13] U Sudeep. Comparisons of tribological and vibration behaviors of textured point contacts of bearing steel lubricated with oil and grease under starved conditions. Journal of Tribology, 2016, 138(3): 1-12.

[14] H Yamada. Numerical and experimental analyses of the dynamic characteristics of journal bearings with square dimples. Journal of Tribology, 2018, 140(1): 1-10.

[15] S Adamczak, P Zmarzły. Research of the influence of the 2D and 3D surface roughness parameters of bearing raceways on the vibration level. Journal of Physics: Conference Series, 2019, 1183 (Conference 1): 012001.

[16] J Yang, Z L Liu, Q C Chen, et al. The effect of wear on the frictional vibration suppression of water-lubricated rubber slat with/without surface texture. Wear, 2019, 426-427(B): 1304-1317.

[17] K Okada, M Yoshida. Copper-graphite brushes lined with porous metals. Electrical Contacts, 1991. Proceedings of the Thirty-Seventh IEEE Holm Conference on. IEEE, 2002: 149-152.

[18] D Shan, Y Han. Static \& dynamic performances analysis and optimization design for the whole casting column of a certain high-speed horizontal machining center using ABAQUS. IEEE International Conference on Information Management and Engineering, 2010: 367-371.

[19] F X Zhou, D Li, X L Cao. Random vibration of fluid-satuated porous elastic plates. Journal of Vibration and Shock, 2017(36): 169-174. (in Chinese)

[20] C S Rajesh, I P Nayan. Impact of various and arbitrary porous structure in the study of squeeze step bearing lubricated with magnetic fluid considering variable magnetic field. Journal of Engineering Tribology, 2015, 229(5): 646-659.

[21] S J Eder, C lelchici, S Krenn, et al. An experimental framework for determining wear in porous journal bearings operated in the mixed lubrication regime. Tribology International, 2018, 123: 1-9.

[22] Yanguo Yin, Guotao Zhang. Lubrication and friction of porous oil bearing materials. IntechOpen, 72620, Chapter 7: 113-122.

[23] S Shitendu, K G Sisir. Comparison between steady-state characteristics of isotropic and anisotropic doubled-layered porous journal bearings under coupled stress lubrication. IOP Conf. Series: Materials Science and Engineering, 2018(377), 012106: 1-6.

[24] N M Bujurke, N B Naduvinamani. On the performance of narrow porous journal bearing lubricated with couple stress fluid. Acta Mechanica, 1991, 86(1-4): 179-191.

[25] S Ohishi. Bearing clearance in design calculation for porousaerostatic thrust bearings. Seimitsu Kogaku Kaishi/Journal of the Japan Society for Precision Engineering, 2018, 84(11): 931-935.

[26] N Sharma. Adiabatic analysis of microtextured porous journal bearings functioned with power law fluid model. Proceedings of the Institution of Mechanical Engineers, Part J: Journal of Engineering Tribology, 2019, 233(10): 1541-1553.

[27] N Sharma, R K Sharma, Sunil, et al. A comparative study for lubrication of surface textured porous journal bearing with two different non-Newtonian fluid models. International Journal of Surface Science and Engineering, 2016, 10(5): 485-502.

[28] ZW Wu, X M Sun, H F Xu, et al. Microstructural characterization and insitu sulfur isotopic analysis of silver-bearing sphalerite from the Edmond hydrothermal field. Central Indian Ridge Ore Geology Reviews, 2018, 92(1): 318-347.

[29] S Lee, D Jang, XY Wang, et al. Lubrication characteristics of a textured porous sliding bearing. Advances in Mechanical Engineering, 2015, 7(3): 404-415.

[30] Y Jin, Z L Liu, Y Z Tian, et al. Vibration monitoring of ship stern bearing based on pulse system. Journal of Wuhan University of Technology, 2010, 32(6): 84-89. (in Chinese) 